\title{
NUMERICAL SIMULATIONS OF GALACTIC OUTFLOW AND INFLOW PHENOMENA
}

\author{
KOHJI TOMISAKA \\ Faculty of Education, Niigata University, \\ Niigata 950-21, JAPAN
}

\begin{abstract}
The recent progress of numerical studies on outflow phenomena from the galactic disk to the halo is summarized. Firstly, a galactic-scale outflow is considered. If the high-velocity cloud is formed from the radiatively cooled gas, which was originally ejected from the disk as a hot gas, the temperature and density at the base of the halo should be $\sim 10^{6} \mathrm{~K}$ and $10^{-3} \mathrm{~cm}^{-3}$. Next, recent results of numerical simulations of the evolution of superbubbles, through which hot gas flows out to the halo, are reviewed. In the case of a thin disk whose density scale height is $H \simeq 100 \mathrm{pc}$, the shell begins to be accelerated upwardly after several dynamical time scales. After that, the polar cap of the shell is broken and the hot gas flows away into the halo. In the case of a thick disk $(H \simeq 500 \mathrm{pc})$ or a magnetized disk with a magnetic field parallel to the disk $(B \simeq 5 \mu \mathrm{G})$, the shell is not accelerated and never shows blow-out.
\end{abstract}

\section{INTRODUCTION}

In recent years, there has been increasing interest in the study of the disk-halo connection, in relation to the structure of the interstellar medium. The volume filling factor of hot tenuous matter, which is essentially heated by supernova (SN) explosions, was estimated to be as high as $f \simeq 75 \%$ from the assumption of global pressure and mass equilibrium (McKee and Ostriker 1977). When their model is applied to the disk and the halo, hot gas spreads over several $\mathrm{kpc}$ above the disk, and the mass exchange rate between the disk and the halo is expected to be $\sim 1 M_{\odot} \mathrm{yr}^{-1}$. Even if the volume filling factor of the hot gas is much smaller than unity, clustered or bunched type II SNe occurring in OB association form superbubbles, through which hot gas heated by the SN explosions can flow into the halo (Norman and Ikeuchi 1989; Heiles 1989). Before (or simultaneously with) the construction of a model of the interstellar medium, it is necessary to study the elementary processes of inflow/outflow phenomena. I summarize in this paper the results of numerical simulations of (1) the global or galactic-scale outflow and (2) the small-scale outflow phenomena, i.e. outflow through superbubbles. 


\section{THE GALACTIC-SCALE OUTFLOW PHENOMENA}

The characteristics of galactic-scale outflow have been studied by Bregman (1980) and Habe and Ikeuchi (1980). The hot gas heated by supernova explosions in the disk flows to the halo. The flow pattern is expected to be as follows: first a hot gas flows upward; with increasing latitude, the gravity becomes weaker; the gas flows radially outward due to the centrifugal force. In this flow, there are two important criteria which determine the flow.

(1) Critical temperature: the gas temperature at which the enthalpy per mass is equal to the effective gravitational potential, i.e., $T_{c}(r) \equiv(\gamma-1) / \gamma \times \mu m_{\mathrm{p}} / k \times\left(|\Phi(r, z=0)|-V_{\phi}^{2} / 2\right)$. Gas in the disk with temperature higher than $T_{c}$ flows away to infinity as a wind and forms Wind-Type Halo.

(2) Cooling time vs. dynamical time: Cooling time scale of the hot gas is estimated as $t_{\text {cool }} \simeq 3 k T /(2 \Lambda n) \simeq 42 \mathrm{Myr}\left(T / 10^{6} \mathrm{~K}\right)^{1.6}\left(n / 10^{-3} \mathrm{~cm}^{-3}\right)^{-1}$. If the radiative cooling does not work so effectively, the dynamical timescale is given as $t_{\mathrm{dyn}} \simeq$ scale height/sound speed $\simeq$ $6.5\left(T / 10^{6} \mathrm{~K}\right)\left(\mathrm{g} / 10^{-8} \mathrm{~cm} \mathrm{~s}^{-2}\right)^{-1} \mathrm{kpc} /\left\{140\left(T / 10^{6} \mathrm{~K}\right)^{1 / 2} \mathrm{~km} \mathrm{~s}^{-1}\right\}$, where $g$ represents the gravitational acceleration in $z$-direction. Comparing these two, $t_{\text {cool }} / t_{\text {dyn }} \sim \mathcal{O}(1)\left(T / 10^{6} \mathrm{~K}\right)^{1.1}$ $\left(n / 10^{-3} \mathrm{~cm}^{-3}\right)^{-1}\left(\mathrm{~g} / 10^{-8} \mathrm{~cm} \mathrm{~s}^{-2}\right)$. In the case of $t_{\text {cool }}<t_{\mathrm{dyn}}$, the outflowing gas is quickly cooled before it reaches the adiabatic scale height, and Cooled-Type Halo is realized. Contrarily, in the case of $t_{\text {cool }}>t_{\text {dyn }}$, the hot gas can not escape from the gravitational potential but can expand into wide region whose size is comparable to the adiabatic scale height. Bound (but not cooled)- Type Halo is formed. These three kinds of flow patterns (Wind-, Bound-, and Cooled-Type Halos) are actually seen in Habe and Ikeuchi (1980).

To explain High Velocity Cloud's (HVC's) by a "fountain model", the clouds must be formed from cooling gas at rather high latitudes, $z \simeq 5-10 \mathrm{kpc}$. This is because in order to achieve a free-fall velocity of $\sim 100 \mathrm{~km} \mathrm{~s}^{-1}$ sufficient distance is necessary. Thus, the most plausible values are $10^{6} \mathrm{~K}$ and $10^{-3} \mathrm{~cm}^{-3}\left(t_{\text {cool }} \simeq t_{d y n}\right)$ (Bregman 1980), which correspond to the values that form the Bound-Type Halo (Habe and Ikeuchi 1980).

\section{ELEMENTARY PROCESSES OF OUTFLOW - THE EVOLUTION OF SUPERBUBBLES}

Clustered type II SNe occurring in OB associations form large-scale supernova remnants called superbubbles. The effect of clustered $\mathrm{SNe}$ was studied in onedimensional hydrodynamics by Bruhweiler et al. (1980) and Tomisaka et al. (1981). It was shown that the size of the bubble exceeds the density scale height in the $z$-direction. More recently, the effect of the density stratification has received much attention. Many two-dimensional hydrodynamical simulations have been done to study the evolution of superbubbles in a stratified medium (Tomisaka et al. 1986; Tenorio-Tagle et al. 1987; Mac Low and McCray et al. 1988; Mac Low et al. 1989; Tenorio-Tagle et al. 1990; Igumentschev et al. 1990). When the size of the bubble exceeds several times the scale height of the disk, the bubble blows 

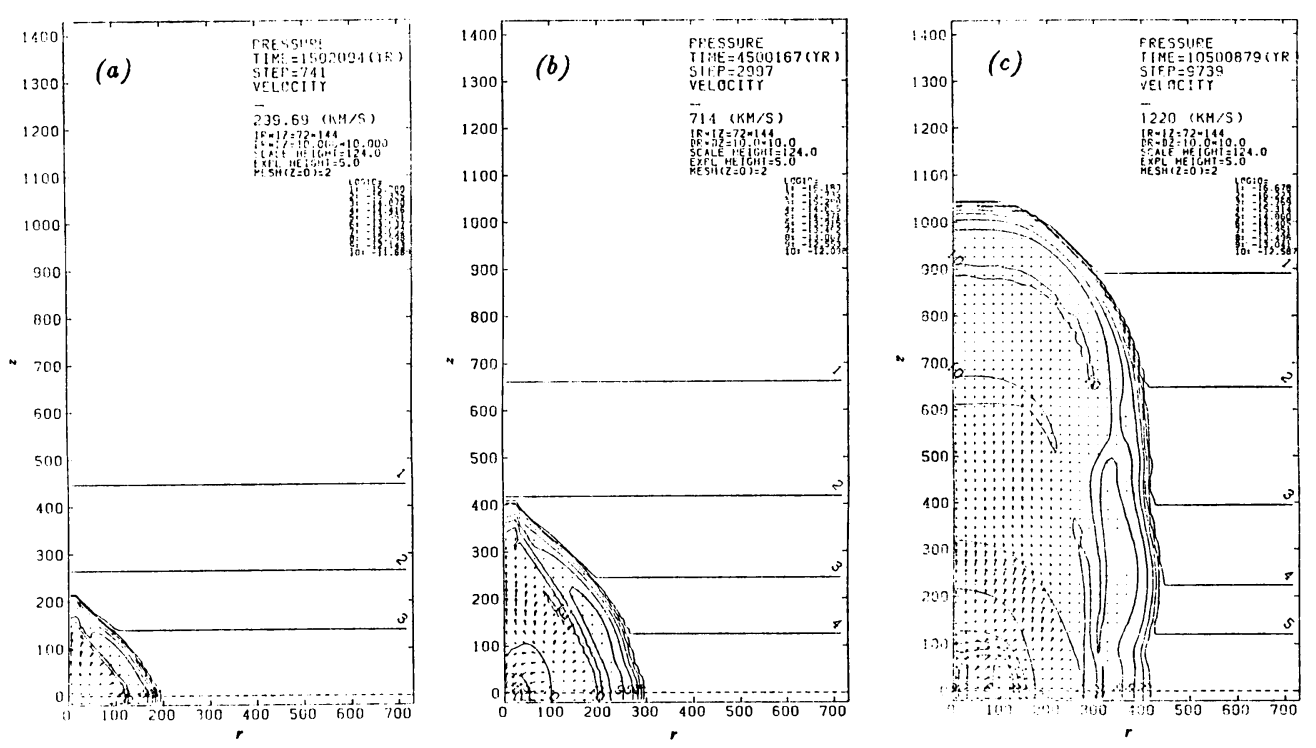

Figure 1. The evolution of a superbubble, with parameters $n_{o}=0.1 \mathrm{~cm}^{-3}$ and $\Delta t=0.2 \mathrm{Myr}$ (Tomisaka and Ikeuchi 1986). The structures at (a) $t=1.5 \mathrm{Myr}$, (b) 4.5 Myr, and (c) 10.5 Myr are shown. It is clear that the spherical bubble is deformed into a funnel shape structure.

out. I will summarize briefly the conclusions of these simulations in the following subsections (for a review see Tenoria-Tagle and Bodenheimer 1988, and Spitzer 1990). Recently, magnetohydrodynamical simulations of magnetized superbubbles were done by Tomisaka (1990) and Shapiro et al. (1990). Magnetic fields parallel to the galactic disk lead to confinement of the bubble in the disk. I will show the recent results of three-dimensional magnetohydrodynamical simulations of a superbubble in $\S 4$.

\subsection{Expansion Law}

There is one characteristic time scale in the dynamics of a bubble in a density stratified medium, which is defined as

$$
\tau_{D} \equiv H^{5 / 3}\left(\frac{\rho_{0}}{L_{\mathrm{SN}}}\right)^{1 / 3} \simeq 1.2 \mathrm{Myr}\left(\frac{H}{100 \mathrm{pc}}\right)^{5 / 3}\left(\frac{\rho_{0}}{2 \times 10^{-24} \mathrm{~g} \mathrm{~cm}^{-3}}\right)^{1 / 3}\left(\frac{L_{\mathrm{SN}}}{10^{38} \mathrm{erg} \mathrm{s}^{-1}}\right)^{-1 / 3}
$$

where $H, \rho_{o}$, and $L_{S N}$ represent, respectively, the density scale height, the density in the mid-plane, and the mechanical luminosity emitted from SN explosions in an $\mathrm{OB}$ association. In case the bubble formed in a spherical symmetric exponential atmosphere, with $\rho=\rho_{o} \exp (-r / H)$, the shock front is decelerated first. The shock 
front and the shell begin to accelerate after $t>2.8 \tau_{D}$, when the size of the shock $R_{s}>2.2 H$ (Kompaneets 1960; Laumbach and Probstein 1969; Koo and McKee 1990).

Figure 1 shows the evolution of a superbubble with parameters $\rho_{o}=2 \times 10^{-25}$ $\mathrm{g} \mathrm{cm}^{-3}$ and $L_{S N}=1.6 \times 10^{38} \mathrm{erg} \mathrm{cm}^{-3}$, using two-dimensional hydrodynamics (Tomisaka and Ikeuchi 1986). Sequential SN explosions with energy $E_{o}=10^{51}$ $\mathrm{erg} / \mathrm{s}$ were assumed to occur every $\Delta \tau \simeq 0.2 \mathrm{Myr}$. However, there is hardly any difference in the global structure between models of continuous energy ejection and sequential SN explosions, if $L_{S N}$ is taken as $E_{o} / \Delta \tau$. It can be seen in Figure 1 that after the size exceeds the scale height $H$, the shell begins to elongate in the $z$-direction. The bubble reaches as high as $z \simeq 1 \mathrm{kpc}$.

The results from a semi-analytical study using the Kompaneets (1960) approximation by Mac Low and McCray (1988) are useful to predict the evolution of a superbubble. In this approximation, the shell is assumed infinitely thin and the pressure distribution in the cavity is assumed uniform. They introduced a nondimensional parameter that represents the ratio of ejected energy in the dynamical time $L_{S N} \tau_{D}$ to the necessary work for the bubble to expand up to the scale height, $p_{e} H^{3}$

$$
\begin{aligned}
D & \equiv\left(\frac{L_{\mathrm{SN}} \tau_{D}}{p_{e} H^{3}}\right)^{3 / 2} \\
& \simeq 940\left(\frac{L_{S N}}{10^{38} \mathrm{erg} \mathrm{s}^{-1}}\right)\left(\frac{H}{100 \mathrm{pc}}\right)^{-2}\left(\frac{p_{e}}{10^{4} k \text { dyne } \mathrm{cm}^{-2}}\right)^{-3 / 2} n_{0}^{-1 / 2}
\end{aligned}
$$

where $p_{e}$ represents the pressure of external interstellar matter. Their numerical results show that, for sufficiently large $D$, the expansion laws of the shell are identical irrespective of $D$ if time is measured in $\tau_{D}$. Furthermore, they showed that in the case of an exponential atmosphere, $\rho(z)=\rho_{o} \exp (-z / H)$, the shell will eventually be accelerated upwardly due to the density stratification, if $D \gtrsim 100$.

\subsection{Rayleigh-Taylor Instability and Blow-out}

Transition from acceleration to deceleration occurs after $(2-3) \times \tau_{D}$ since the SN explosions started (Table 1). Small differences seem to come from the adopted initial density distribution. What happens in the upwardly accelerated shell? In a frame co-moving with the accelerated shell, the effective gravity (really an inertia force) works downwards. The cooled shell is pushed by tenuous hot gas against gravity. This configuration is unstable for Rayleigh-Taylor instability (Różyszka and Tenorio-Tagle 1985). The instability grows very rapidly after the transition, and finally the shell begins to break at the polar cap. This is clearly seen in numerical simulations by Tomisaka and Ikeuchi (1987), Mac Low et al. (1989), and Tenorio-Tagle et al. (1990). 
TABLE 1.

Transition from Deceleration to Acceleration.

\begin{tabular}{llccr}
\hline \hline Authors & Initial Density Distribution & Transition Time $\tau_{T}$ & Height at $\tau_{T}$ & $n_{0}$ \\
\hline $\mathrm{TI}^{a}$ & Fuchs \& Thielheim atmosphere & $\simeq 2 \tau_{D}$ & $\simeq 2 \mathrm{H}$ & 0.1 \\
$\mathrm{MMN}^{b}$ & exponential atmosphere & $3.3 \tau_{D}$ & $2.9 H$ & 1 \\
& Gaussian atmosphere & $2.5 \tau_{D}$ & $1.7 \mathrm{H}$ & 1 \\
\hline
\end{tabular}

${ }^{a}$ Tomisaka and Ikeuchi (1986).

${ }^{b}$ Mac Low et al. (1989).

\subsection{Effect of a Thick Disk}

The acceleration of the shell and the Rayleigh-Taylor instability at the polar cap may be typical for a superbubble in a thin disk with a scale height of $H \simeq 100 \mathrm{pc}$. There is good observational evidence, however, for a warm HI component whose density scale height is $\sim 500 \mathrm{pc}$ (Lockman et al. 1986). The transition time $\tau_{T}$ is given by

$$
\tau_{T} \simeq 30 \mathrm{Myr}\left(\frac{L_{S N}}{10^{38} \mathrm{erg} \mathrm{s}^{-1}}\right)^{-1 / 3}\left(\frac{H}{500 \mathrm{pc}}\right)^{5 / 3}\left(\frac{n_{0}}{0.1 \mathrm{~cm}^{-3}}\right)^{1 / 3}
$$

The duration of an active phase of $\mathrm{SN}$ explosions in an $\mathrm{OB}$ association is estimated to be 30-50 Myr (from the initial mass function and stellar lifetime data). It can be concluded that the shell of a superbubble is decelerated throughout its lifetime. Actually, from the numerical results presented by Tenorio-Tagle et al. (1990), blow-out as well as acceleration is not seen in the model with an exponential atmosphere with $H=500 \mathrm{pc}$ and $\rho_{o}=1.6 \times 10^{-25} \mathrm{~g} \mathrm{~cm}^{-3}$ in $10 \mathrm{Myr}$. However, the superbubble expands and still reaches $z \simeq 1 \mathrm{kpc}$ during its lifetime.

\section{MAGNETIZED SUPERBUBBLES}

The flow across a magnetic field is blocked in case of a frozen-in field. Therefore, as in the case of a thick disk, the magnetic field tends to prevent expansion of the superbubble in the $z$-direction, if it runs parallel to the galactic disk. Moreover, there is a possibility that the magnetic field completely confines the superbubble in the galactic disk. In addition, in a magnetized interstellar medium, hydrodynamical shock waves are replaced by magnetohydrodynamical (MHD) shock waves. In this case, the fast MHD shock wave propagates and compresses the magnetic-field. This section presents recent results on the evolution of magnetized superbubbles. The strength of the magnetic field $B$ is not well determined observationally; it is 
TABLE 2.

Density Distribution.

\begin{tabular}{|c|c|c|c|}
\hline Component & $\begin{array}{c}\rho(0)^{a} \\
\left(10^{-25} \mathrm{gcm}^{-3}\right)\end{array}$ & $\begin{array}{l}H^{b} \\
(\mathrm{pc})\end{array}$ & Distribution \\
\hline Cold HI .. & 6.8 & 135 & Gaussian $^{c}$ \\
\hline Warm Hi ....... & 1.8 & 135 & Gaussian \\
\hline Warm HI ....... & 2.3 & 400 & exponential ${ }^{d}$ \\
\hline $\mathrm{H}_{2} \ldots \ldots \ldots \ldots$ & 6.8 & 70 & Gaussian \\
\hline Warm Hil ....... & 0.56 & 1000 & exponential \\
\hline \multicolumn{4}{|c|}{ a Density on the $z=0$ plane. } \\
\hline \multicolumn{4}{|c|}{ bensity scale-height. } \\
\hline \multicolumn{4}{|c|}{${ }^{c} \rho=\rho(0) \exp \left(-z^{2} / 2 H^{2}\right)$. } \\
\hline
\end{tabular}

reported to $\sim 5 \mu \mathrm{G}$ in the warm ionized interstellar matter (Rand and Kulkarni 1989). Magnetic pressure, $p_{m a g} \simeq 10^{-12}(B / 5 \mu \mathrm{G})^{2}$ dyne $\mathrm{cm}^{-2}$, cannot be ignored in comparison with the ram pressure exerted on the superbubble shell, $p_{\text {ram }} \simeq$ $10^{-12}\left(\rho / 2 \times 10^{-24} \mathrm{~g} \mathrm{~cm}^{-3}\right)\left(V / 10 \mathrm{~km} \mathrm{~s}^{-1}\right)^{2}$ dyne $\mathrm{cm}^{-2}$, where $\rho$ and $V$ represent the ambient density and expansion speed of the shell.

\subsection{Model}

It is assumed that the interstellar medium in which the $\mathrm{SN}$ begin to explode is in hydrostatic equilibrium. A realistic hydrostatic model similar to that of Bloemen (1987) is taken, i.e., five gas components (Table 2), supported by thermal pressure against gravity $g_{z}(z)$ (Bahcall 1984)

$$
p_{t h}(z)+p_{m a g}(z)=\int_{0}^{z} \rho(z) g_{z}(z) d z+p(0)
$$

where $p(0)$ is the total (thermal + magnetic) pressure at $z=0$, taken to be $3 \times 10^{-12}$ dyne $\mathrm{cm}^{-1}$. Since the scale-height of the magnetic field strength is much larger than that of the density distribution, the strength of the magnetic field is assumed uniform irrespective of $z$. Further, I assume that the OB association is located in the $z=0$ plane. The mechanical luminosity emitted by SN explosions is given by $L_{S N} \simeq 1.68 \times 10^{38}\left(E_{o} / 10^{51} \mathrm{erg}\right)\left(\Delta \tau / 2 \times 10^{5} \mathrm{yr}\right)^{-1} \mathrm{erg} / \mathrm{s}$.

Cartesian coordinates are adopted: the $x$-axis is chosen as the direction of the initial magnetic field and the $z$-axis is chosen perpendicular to the galactic disk ( $x-$ $y$ plane). Since both the density stratification and the magnetic field parallel to the galactic disk are included, the problem to be solved becomes a three-dimensional MHD flow. The "monotonic scheme" (van Leer 1977; Norman and Winkler 1986) is adopted to solve the hydrodynamical equations and the "constrained transport method" (Evans and Hawley 1988) is used to solve the induction equation of the magnetic field. The number of meshes is $81^{3}$ or $81^{2} \times 121$ in Cartesian coordinates $(x, y, z)$. The amount of memory required is $128-192 \mathrm{MB}$. Since the code of this 
finite-difference scheme is totally vectorized, only $0.7-1$ second of CPU time is needed for each time step using the HITACHI S-820/80 supercomputer at the University of Tokyo.

Sequential SN explosions were replaced by a continuous energy release, as was done by Mac Low et al. (1989), and the gas was assumed adiabatic with $\gamma=5 / 3$ for simplicity. Since the amount of the energy lost by radiative cooling has not been determined definitely (Mac Low et al. 1989; Tomisaka and Ikeuchi 1986), I studied two cases with $L_{S N}=1.68 \times 10^{37} \mathrm{erg} / \mathrm{s}$ and $L_{S N}=1.68 \times 10^{38} \mathrm{erg} / \mathrm{s}$ to make up for the adiabatic assumption.

\subsection{Numerical Results}

Figure 2 shows the structure of the superbubble, $10 \mathrm{Myr}$ after the SN explosions began. The structure is similar to that of the non-magnetic bubble, i.e., the matter ejected from the SNe flows outward as a free wind; ejected matter passes through the inward-facing shock; the accumulated interstellar matter and the magnetic field are spread between the contact surface and the outward-moving MHD shock. The outward-moving wave front is seen at $x \simeq 350 \mathrm{pc}$ (Fig. $2 \mathrm{a}$ and c), $y=400 \mathrm{pc}$ (Fig. $2 \mathrm{c})$. In the $z$-direction, it has already reached the upper boundary $z_{\max }=800 \mathrm{pc}$. The hot gas is distributed over the region where the velocity vectors are plotted in Figure 2. From Figures $2 a$ and b, it can be seen that the hot matter formed by SN explosions is confined below $z \lesssim 200$ pc. The magnetic field is swept up and compressed in the $z \gtrsim 200$ pc region by the thermal pressure of the hot matter below. Figure 2c clearly shows that the hot matter does not extend much in the $y$-direction $(y \lesssim 150 \mathrm{pc}$ ) and that the flow is focused in the $x$-direction. The outer boundary of the hot matter (contact surface) expands as $\left(x_{i}, y_{i}, z_{i}\right) \simeq(160 \mathrm{pc}, 130$ $\mathrm{pc}, 150 \mathrm{pc})^{1}$ at $5 \mathrm{Myr},(200 \mathrm{pc}, 140 \mathrm{pc}, 180 \mathrm{pc})$ at $7.5 \mathrm{Myr}$, and $(220 \mathrm{pc}, 140$ pc, $200 \mathrm{pc}$ ) at $10 \mathrm{Myr}$. Compared with the model of no magnetic field but the same density distribution, in which the hot gas extends up to $\left(x_{i}, y_{i}, z_{i}\right) \simeq(170$ pc, $170 \mathrm{pc}, 290 \mathrm{pc}$ ) in $t=10 \mathrm{Myr}$ (Figure 3), for the model I have studied it can be concluded that the magnetic field dams the flow across the field line and encourages the flow parallel to the field line ( $x$-direction). The hot gas is confined within $z \lesssim 200 \mathrm{pc}$ by the magnetic field. Outside the hot matter, warm gas with $T \lesssim 10^{4} \mathrm{~K}$, heated by the outermost wave front, is distributed in a rather thick region $(250 \mathrm{pc}<x<400 \mathrm{pc}, 130 \mathrm{pc}<y<450 \mathrm{pc}, z>200 \mathrm{pc})$. The present simulation is restricted to the adiabatic process; in reality, due to radiative cooling, a cooled thin shell will replace this warm gas and surround the hot gas. Although the magnetic field is swept and compressed in this warm region, thermal pressure predominates over the magnetic pressure. Extrapolating the expansion law of $x_{i}$, the hot gas occupies the region with $z_{i} \lesssim 300$ pc during the active phase of the OB association ( $30 \mathrm{Myr})$.

1 This expression means that the boundary crosses the $x$-axis at $\left(x_{i}, 0,0\right), y$-axis at $\left(0, y_{i}, 0\right)$, and so on. 

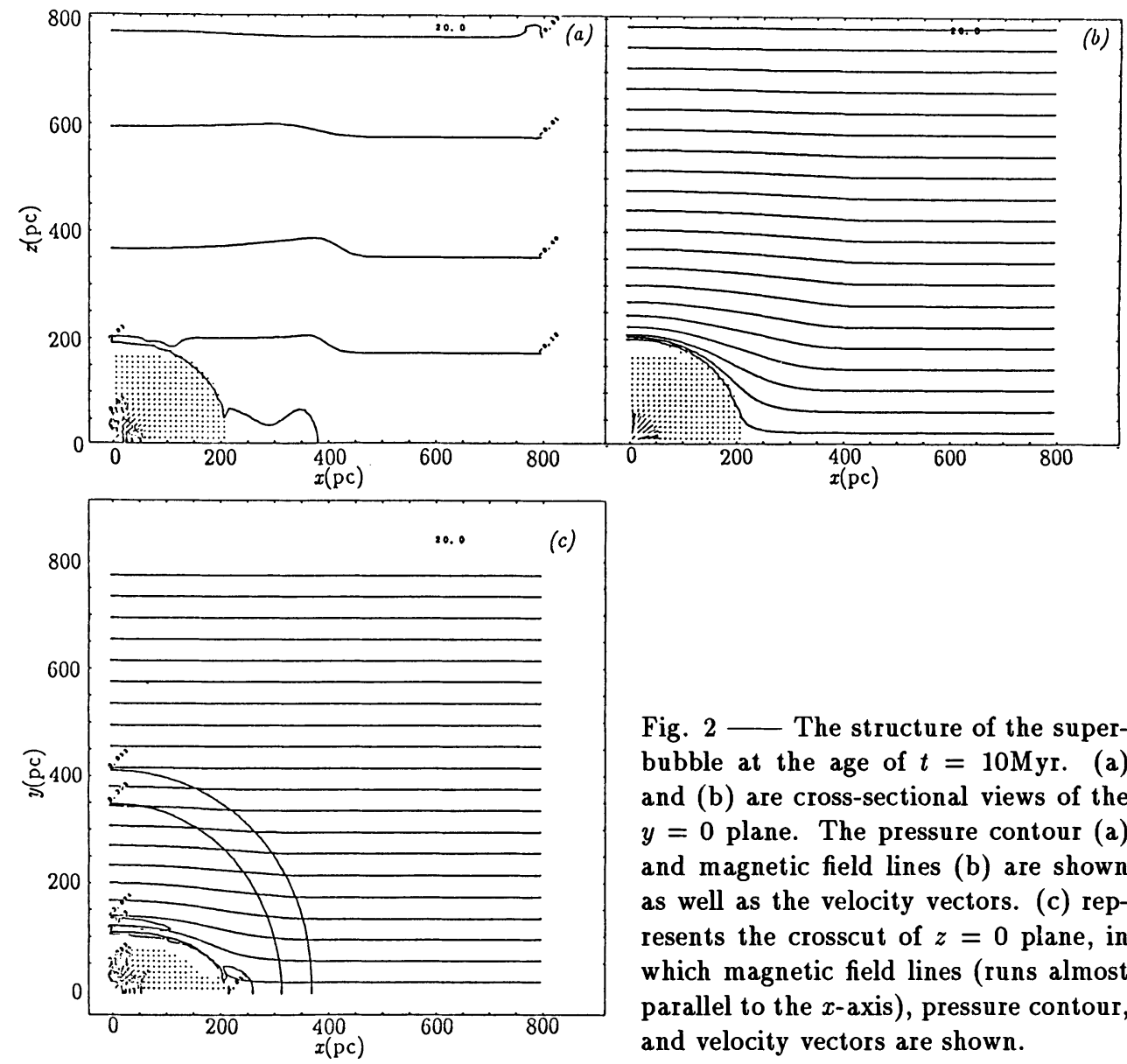

Fig. $2-$ The structure of the superbubble at the age of $t=10 \mathrm{Myr}$. (a) and (b) are cross-sectional views of the $y=0$ plane. The pressure contour (a) and magnetic field lines (b) are shown as well as the velocity vectors. (c) represents the crosscut of $z=0$ plane, in which magnetic field lines (runs almost parallel to the $x$-axis), pressure contour, and velocity vectors are shown.

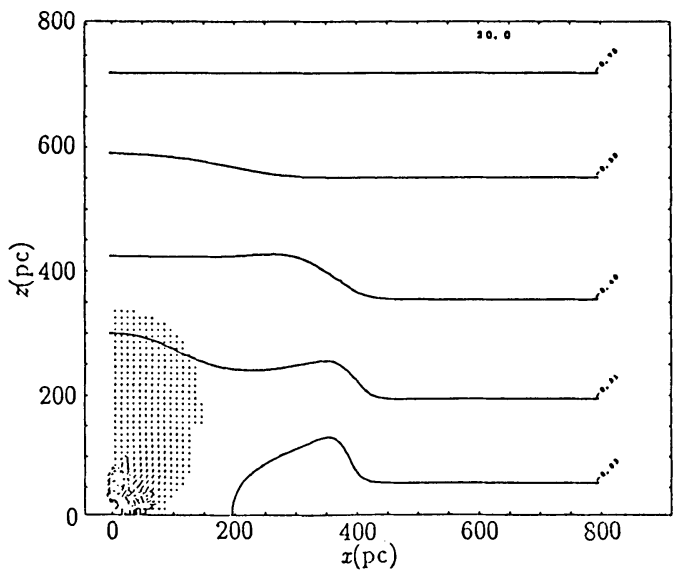

Fig. 3 The same as Figure 2a but for the case with no magnetic field but the same density distribution. 


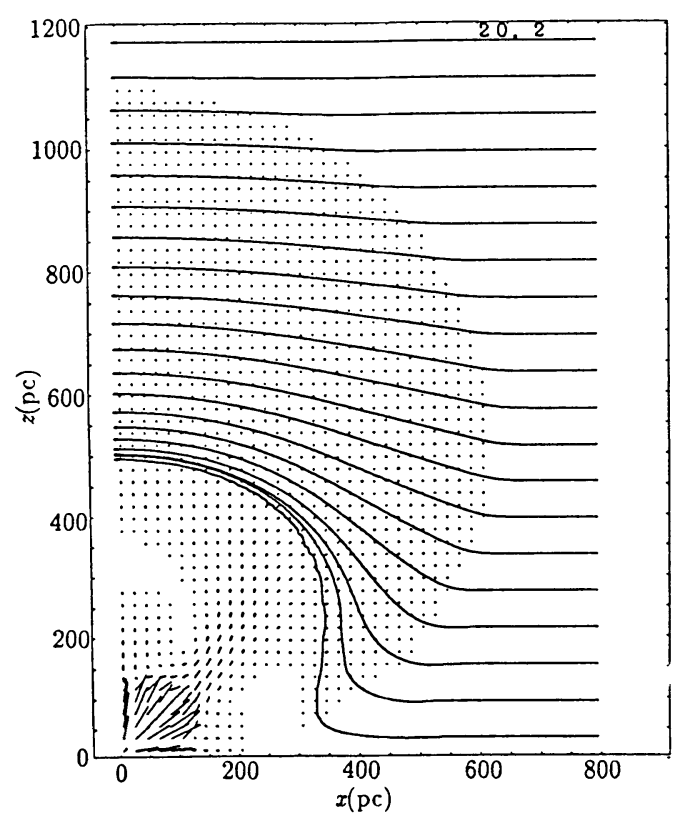

Fig. 4 The structure of the superbubble at the age of $t=10 \mathrm{Myr}$ for the case with the mechanical luminosity ten times larger than the previous case.

Expansion is highly anisotropic, i.e., the outermost wave front expands as $\left(x_{s}, y_{s}, z_{s}\right) \simeq$ $(220 \mathrm{pc}, 230 \mathrm{pc}, 310 \mathrm{pc})$ at $5 \mathrm{Myr},(300 \mathrm{pc}, 320 \mathrm{pc}, 520 \mathrm{pc})$ at $7.5 \mathrm{Myr}$, and $(370 \mathrm{pc}$, $410 \mathrm{pc},>800 \mathrm{pc})$ at $10 \mathrm{Myr}$. Although the height of the hot-warm interface is decelerated as $z_{\mathrm{i}} \propto t^{0.4}$, that of the outermost wave front is accelerated. Acceleration of $z_{\mathrm{s}}$ is mainly driven by steep density gradient $\partial \rho / \partial z$. Perpendicularly to the magnetic field ( $y$ - and $z$ direction) the expansion is encouraged by the magnetic field, since in these directions waves propagate as the magnetosonic wave mode (Kulsrud et al. 1965). This encouragement by the magnetic field can be seen by comparing this model with that of no magnetic field but the same density distribution (Figure 3$)$, in which the size is as large as $\left(x_{s}, y_{s}, z_{s}\right) \simeq(360$ $\mathrm{pc}, 360 \mathrm{pc}, 570 \mathrm{pc})$ at $10 \mathrm{Myr}$.

In Figure 4, I plot the structure of the superbubble at the age of $10 \mathrm{Myr}$ with $L_{\mathrm{SN}}=$ $1.68 \times 10^{38} \mathrm{ergs}^{-1}$, which is ten times larger than the previous case. It is interesting that the MHD shock seen in $z \lesssim 500 p c$ is so called "intermediate shock." Due to the large luminosity, the superbubble expands much faster: $\left(x_{\mathrm{s}}, y_{\mathrm{s}}, z_{\mathrm{s}}\right) \simeq(470 \mathrm{pc}, 500 \mathrm{pc}, 1100 \mathrm{pc})$ at $10 \mathrm{Myr}$. Since the pressure of the of the inner hot gas is high, also the hot-warm interface expands faster than that of the previous case: $\left(x_{\mathrm{i}}, y_{\mathrm{i}}, z_{\mathrm{i}}\right) \simeq(320 \mathrm{pc}, 200 \mathrm{pc}, 500 \mathrm{pc})$. It is clear that before the typical lifetime of massive stars in $\mathrm{OB}$ associations the hot gas in the bubble reaches the halo region $(z \gtrsim 1 \mathrm{kpc})$. From this result, it is concluded that if the radiative cooling is inefficient, the magnetic field assumed here $\sim 5 \mu \mathrm{G}$ cannot prevent the hot matter from flowing out to the halo.

From the study of spherical symmetric nonmagnetic wind-blown bubble (Weaver et al. 1976), the pressure in the shocked wind (ejected matter) region is expressed as

$$
p \simeq 0.17\left(L_{W}^{2} \rho_{0}^{3}\right)^{1 / 5} t^{-4 / 5},
$$

and the expansion of contact surface between the shocked wind and accumulated matter is

$$
R_{i} \simeq 0.76\left(L_{W} / \rho_{0}\right)^{1 / 5} t^{3 / 5}
$$


where $L_{W}$ represents the mechanical luminosity of wind $=L_{S N}$. If the structure of the magnetic bubble is not far from that of the non-magnetic one, when $p \simeq p_{\text {mag }}$, the radius of the contact surface reaches

$$
\begin{aligned}
\bar{R}_{i} & \simeq 2.22 L_{W}^{1 / 2} \rho_{0}^{1 / 4} B_{0}^{-3 / 2} \\
& \simeq 230 \mathrm{pc}\left(L_{W} / 1.68 \times 10^{37} \mathrm{erg} \mathrm{s}^{-1}\right)^{1 / 2}\left(\rho_{0} / 1.8 \times 10^{-24} \mathrm{~g} \mathrm{~cm}^{-3}\right)^{1 / 4}\left(B_{0} / 6 \mu \mathrm{G}\right)^{-3 / 2}
\end{aligned}
$$

If $\bar{R}_{i} \gg H$, before magnetic field works, due to the density stratification the superbubble breaks through the disk (the model shown in Figure 4: $L_{S N}=1.67 \times 10^{38} \mathrm{erg} \mathrm{s}^{-1}$ ). On the other hand, if $\bar{R}_{i} \lesssim H$, before density stratification works, the superbubble is confined by the magnetic field (the model shown in Figure 2: $L_{S N}=1.67 \times 10^{37} \mathrm{erg} \mathrm{s}^{-1}$ ).

This work was partially supported by Grant-in-Aid for Encouragement of Young Scientists from the Ministry of Education, Science, and Culture (02854016). I would like to express my gratitude to Yamada Science Foundation and IAU for their travel support.

\begin{tabular}{|c|l|l|}
\hline \hline & Thin Disk $H \sim 100 \mathrm{pc}$ & Thick Disk $H \gtrsim 500 \mathrm{pc}$ \\
\hline$B=0$ & $\begin{array}{l}\text { Accelerated. Broken at } \\
\text { the Polar Cap. }\end{array}$ & $\begin{array}{l}\text { Not Accelerated. Reach } \\
z \sim 1 \mathrm{kpc} .\end{array}$ \\
\hline$B \sim 5 \mu \mathrm{G}$ & & $\begin{array}{l}\text { Not Accelerated. Much } \\
\text { Confined. }\end{array}$ \\
\hline
\end{tabular}

summary

\section{REFERENCES}

Bahcall, J. N. 1984, Ap. J., 276, 169.

Bloemen, J. B. G. M. 1987, Ap. J., 322, 694.

Bregman, J. N. 1980 Ap. J. 236, 577.

Bruhweiler, F. C., Gull, T. R., Kafatos, M., and Sofia, S. 1980, Ap. J. (Letters), 238, L27.

Cash, W., Charles, P. Bowyer, S., Walter, F., Gamire, G., and Riegler, G. 1980, Ap. J. $238, \mathrm{L71}$.

Evans, C. R., and Hawley, J. F. 1988, Ap. J., 332, 659.

Habe, A., and Ikeuchi, S. 1980, Prog. Theor. Physics, 64, 1955.

Heiles, C. 1989, in Structure and Dynamics of the Interstellar Medium, eds. by G. Tenorio-

Tagle, M. Moles, and J. Melnick (Berlin: Springer), p. 484.

Igumentshchev, I. V., Shustov, B. M., and Tutukov, A. V. 1990 Astr. Ap. in press.

Kompaneets, A. S. 1960, Soviet Phys. Dokl., 5, 46.

Koo, B.-C., and McKee, C. F. 1990, Ap. J., 354, 513.

Kulsrud, R. M., Berstein, I. B., Kruskal, M., Fanussi, J., and Ness, N. 1965, Ap. J., 142, 491.

Laumbach, D. D., and Probstein, R. F. 1969, J. Fluid Mech., 35, 53.

Lockman, F. J., Hobbs, L. M., and Shull, J. M. 1986, Ap. J, 301, 380.

Mac Low, M.-M., and McCray, R. 1988, Ap. J., 324, 776.

Mac Low, M.-M., McCray, R. and Norman, M. L. 1989, Ap. J., 337, 141.

McKee, C. F., and Ostriker, J. P. 1977, $A p$. J, 218, 148.

Norman, C. A., and Ikeuchi, S. 1989, Ap. J., 345, 372.

Norman, M. L., and Winkler, K.-H. A. 1986, in Astrophysical Radiation Hydrodynamics, eds. by K.-H. A. Winkler and M. L. Norman (Dordrecht: Reidel), p. 187.

Rand, R. J., and Kulkarni, S. R. 1989, Ap. J., submitted.

Reynolds, R. J., and Ogden, P. M. 1979, Ap. J., 229, 942.

Różyczka, M., and Tenorio-Tagle, G. 1985, Astr. Ap., 147, 209.

Shapiro, P. R., Mineshige, S., and Shibata, K. 1990, this volume.

Spitzer, L., Jr. 1990, Ann. Rev. Astr. Ap. in press.

Tenorio-Tagle, G., Bodenheimer, P., and Różyczka, M. 1987, Astr. Ap., 182, 120.

Tenorio-Tagle, G., and Bodenheimer, P. 1988, Ann. Rev. Astr. Ap., 26, 145.

Tenorio-Tagle, G., Różyczka, M., and Bodenheimer, P. 1990, submitted to Astr. Ap..

Tomisaka, K. $1990 \mathrm{Ap}$. J. (Letters) in press.

Tomisaka, K., and Ikeuchi, S. 1986, Pub. Astr. Soc. Japan, 38, 697. . 1987, Ap. J., 330, 695 .

Tomisaka, K., Habe, A., and Ikeuchi, S. 1981, Ap. Space Sci., 78, 273.

van Leer, B. 1977, J. Comput. Phys., 23, 276.

Weaver, R., McCray, R., Castor, J., Shapiro, P., and Moore, R. 1977, Ap. J., 218, 377; $220,742$. 